\title{
The prevalence of sodium abnormalities in moderate to severe traumatic brain injury patients in a level 1 Trauma unit in Durban
}

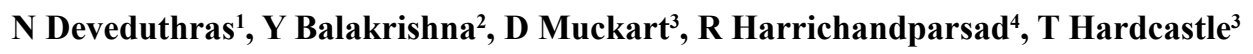 \\ ${ }^{1}$ Neurosurgery Registrar, Department of Neurosurgery (IALCH \& UKZN), Inkosi Albert Luthuli Central Hospital \\ ${ }^{2}$ MRC Biostatistics Unit, 491 Ridge Road, Durban \\ ${ }^{3}$ Trauma service IALCH \& Department of Surgery UKZN, Inkosi Albert Luthuli Central Hospital \\ ${ }^{4}$ Department of Neurosurgery (IALCH \& UKZN), Inkosi Albert Luthuli Central Hospital
}

Corresponding author: Dr Nikesh Deveduthras (Nikeshd4@gmail.com)

\begin{abstract}
Background: The prevalence of sodium abnormalities in the moderate to severe brain injury patient is not known in the South African population.

Objectives: Determine the prevalence of sodium abnormalities in moderate to severe traumatic brain injury. Determine Glasgow Outcome Score (GOS) between sodium groups.

Methods: Patients admitted to the trauma intensive care unit between January 2013 and June 2015 with moderate to severe traumatic brain injury were included in the study. Descriptive statistics, tests of association and tests of differences were used.

Results: There were 184 patients with 143 (77.7\%) males and 41 (22.3\%) females. Abnormal sodium was present in $126(68.4 \%), 61$ of whom had hyponatremia and 65 hypernatremia, a prevalence of $33.1 \%$ and $35.3 \%$ respectively. Of the 65 patients with hypernatremia, $52(80 \%)$ had dehydration, $7(10.7 \%)$ had diabetes insipidus (DI) and $6(9.3 \%)$ had hyperosmolar therapy as the cause. Of the 61 patients with hyponatremia, the commonest cause was fluid overload in 47 patients $(77.1 \%)$ with SIADH in $11(18 \%)$ and CSWS in $3(4.9 \%)$. Death occurred in $34(18.5 \%)$ patients and diagnosis was found to be significantly associated with mortality $(\mathrm{p}=0.01)$, the most common diagnoses amongst those who died being dehydration (29.4\%), fluid overload (17.7\%) and DI (14.7\%).

Conclusions: The prevalence of sodium abnormalities was $126(68.4 \%)$ patients of whom $61(33.1 \%)$ had hyponatremia and $65(35.3 \%)$ hypernatremia. In those patients who survived, a later onset was related to a better outcome. The GOS in DI tended to be worse.

Keywords: Sodium abnormalities, Moderate to severe traumatic brain injury, Hypernatremia Hyponatremia, Diabetes insipidus (DI), Glasgow Outcome Score (GOS), Cerebral salt wasting (CSW)
\end{abstract}

S Afr J Surg 2019;57(2)

http://dx.doi.org/10.17159/2078-5151/2019/v57n2a2823

\section{Introduction}

\section{What is known}

Traumatic brain injury (TBI) accounts for $50 \%$ of deaths from bodily injury and of those who survive many suffer long-term functional disability. ${ }^{1}$ As the vast majority of trauma patients are children or young adults, the socio-economic effects are of major consequence. Little can be done to correct the primary damage to the central nervous system and management must focus on preventing secondary insults. Of critical importance is the prevention of raised intracranial pressure and reduced cerebral perfusion as a result of brain oedema. Meticulous attention should be paid to fluid administration and fluid balance..$^{2,3,4}$ Sodium, the major extracellular cation, plays a critical role in fluid balance and abnormalities are not uncommon in the moderate to severe traumatic brain injury population. ${ }^{4,5,6}$. As a result of its osmotic activity, both elevated and decreased abnormalities in serum concentrations have the potential to cause severe secondary brain injury. ${ }^{6}$

\section{What is not known}

The prevalence of sodium abnormalities in the moderate to severe brain injury patient is not known in the South African population. The diagnosis of sodium abnormality in this cohort of patients has not been previously studied with most studies being performed in ICU patients with multiple comorbidities in other countries. ${ }^{13,15}$ The relationship between CT findings and sodium abnormality has not previously been assessed in our population. The relationship between sodium abnormality, diagnosis and outcome has not been studied previously in isolated brain injury patients in our setting. It is not known if the onset of sodium abnormality has any effect on the outcome of the patient. 


\section{What is being studied}

The main aim of the study was to determine the prevalence of sodium abnormalities in the moderate to severe brain injury patient in the Durban population. The ancillary aim of this study was to determine the aetiology of sodium abnormalities in patients suffering TBI and the relationship to outcome, and to look at the onset of sodium abnormality and the effect on outcome.

\section{Methods}

This retrospective descriptive study was conducted on patients who were admitted to the Level I Trauma Unit at Inkosi Albert Luthuli Central Hospital (IALCH) in Durban, South Africa, between January 2013 and June 2015. The study protocol was approved by the Biomedical Research Ethics Committee of the University of KwaZulu-Natal, BREC study number BE008/16 (sub-study of BCA207/09). The aim of the study was to determine the prevalence and aetiology of sodium abnormalities in patients with moderate to severe brain injury admitted to the trauma unit.

Data were extracted from the hospital software system for clinical records (Sorian ${ }^{\mathrm{TM}}$, Siemens, Germany) and the prospectively maintained trauma unit clinical database. All patients were managed as per ATLS principles and underwent a neurosurgical examination and computed tomography of the brain. Only moderate (GCS $9-13$ ) and severe (GCS < 9) head injury patients were considered in the study as these are the patients commonly admitted to our trauma ICU and patients with a better GCS can control their fluid intake through normal physiologic mechanisms. Excluded from the study were all patients with multiple compartment injuries, renal disease or acute kidney injury (AKI - KDIGO Grade 2 to 3), cardiac disease and prior sodium disorders. The first blood sample was obtained on admission to the resuscitation area of the trauma unit and thereafter daily. Patients were defined according to the sodium abnormality and classified as having normal sodium concentrations, hypernatremia or hyponatremia. Age, sex, mechanism of injury, CT findings, surgical management, onset of sodium abnormality, duration, peak, and trough levels were recorded. Outcome was determined by the Glasgow Outcome Scale (GOS).

Definitions: Normal serum sodium was defined as levels between $135 \mathrm{mmol} / \mathrm{L}$ to $144 \mathrm{mmol} / \mathrm{l}$. Hyponatremia was defined by a serum concentration $<135 \mathrm{mmol} / \mathrm{l}$ and hypernatremia $>145 \mathrm{mmol} / \mathrm{l}$. The sodium abnormalities were graded into mild, moderate and severe hyper- or hyponatremia as follows. Severe hypernatremia was defined as a serum sodium of $160 \mathrm{mmol} / \mathrm{L}$ or greater, moderate hypernatremia as a serum sodium from $155-159 \mathrm{mmol} / \mathrm{L}$, mild hypernatremia as a serum sodium from $150 \mathrm{mmol} / \mathrm{L}$ to $154 \mathrm{mmol} / \mathrm{L}$, mild hyponatremia as a serum sodium less than $135 \mathrm{mmol} / \mathrm{L}$, moderate hyponatremia as a serum sodium less than $130 \mathrm{mmol} / \mathrm{L}$ and severe hyponatremia as a serum sodium less than $125 \mathrm{mmol} / \mathrm{L}$. Hypernatremic patients were classified into those with suspected diabetes insipidus (DI), dehydration or secondary to hypertonic saline use. Those with hyponatremia were classified into suspected fluid overload, Syndrome of inappropriate $\mathrm{ADH}$ secretion (SIADH), and Cerebral Saltwasting Syndrome(CSWS). DI was defined as polyuric hypernatremia with a serum osmolarity $>280 \mathrm{mosm} / \mathrm{l}$ and urine osmolarity $<200 \mathrm{mosm} / \mathrm{l}$. Dehydration was defined as hypernatremia with serum osmolarity $>280 \mathrm{mosm} / 1$ and urine osmolarity normal or high. SIADH was defined as hypotonic hyponatremia with a serum osmolarity $<275 \mathrm{mosm} / 1$ and an inappropriately high urine osmolarity $>100 \mathrm{mosm} / \mathrm{l}$ with either euvolemia or hypervolemia. CSWS was diagnosed by hyponatremia, dehydration with an elevated serum osmolarity and a urine sodium $>20 \mathrm{mEq} / \mathrm{L}$.

Statistical analysis: Data were analysed using Stata 14 (StataCorp, College Station, TX, USA). Continuous variables were described using either means (standard deviation - SD) or medians (interquartile ranges - IQR). Categorical variables were described as frequencies and percentages. Pearson's Chi-squared test or Fisher's exact test, where applicable, were used to assess associations between categorical variables and sodium abnormality. Differences in continuous variables between groups was determined using the Kruskal-Wallis test. Spearman's correlation coefficient was also calculated. A p-value less than 0.05 was considered statistically significant.

\section{Results}

Of 803 admissions during the study period 184 (22.9\%) had either isolated TBI or TBI with concomitant minor musculoskeletal injury. Males accounted for $143(77.7 \%)$ admissions with a male to female ratio of $3.5: 1$. The median age was 29 years (IQR 13.5 - 36). Forty-one (22.3\%) patients were children (defined as less than 13 years of age). Motor vehicle related collisions accounted for $71.2 \%$ of injuries. Less common were blunt assaults (9.7\%), falls from a height $(6 \%)$, penetrating assaults $(3.8 \%)$, found unconscious $(9.3 \%)$. The population characteristics, sodium abnormalities, and outcome in relationship to severity of TBI are tabulated in Tables 1-3.

Table 4 illustrates TBI pathology as determined by CT scanning. Diffuse brain injury occurred in $39.7 \%$, cerebral contusions in $16.3 \%$, acute subdural haematoma in $15.2 \%$, traumatic subarachnoid haemorrhage in $4.9 \%$, intraventricular haemorrhage in $2.2 \%$ of patients, acute subdural haematoma with contusions in $5.4 \%$, acute extradural haematoma in $6 \%$, brainstem elongation in $0.5 \%$, tract haematoma in $1.6 \%$, intracerebral haematoma in $1.1 \%$, subacute subdural haematoma in $0.5 \%$, posterior fossa extradural haematoma in $0.5 \%$, comminuted skull fracture in $4 \%$, posterior fossa contusion in $0.5 \%$, skull base fracture in $1.1 \%$, previous infarct with no new haemorrhage in $0.5 \%$.

Patients were treated as follows: 67 (36.4\%) having only an ICP monitor, $38(20.7 \%)$ had a craniotomy and an ICP monitor, 27 (14.7\%) had a decompressive craniectomy and an ICP monitor, 49 (26.6\%) had neuroprotective ventilation only, with no ICP monitor inserted, $2(1.1 \%)$ had a wound debridement and $1(0.5 \%)$ was admitted for neurological observation. 


\begin{tabular}{|c|c|c|c|c|c|}
\hline Characteristic & $\begin{array}{l}\text { Hyponatremia } \\
(\mathrm{n}=61)\end{array}$ & $\begin{array}{l}\text { Normal } \\
(n=58)\end{array}$ & $\begin{array}{l}\text { Hypernatremia } \\
\qquad(\mathrm{n}=65)\end{array}$ & $\begin{array}{c}\text { Total } \\
(n=184)\end{array}$ & p-value \\
\hline Gender & & & & & 0.708 \\
\hline Male & $49(80.3)$ & $43(74.1)$ & $51(78.5)$ & $143(77.7)$ & \\
\hline Female & $12(19.7)$ & $15(25.9)$ & $14(21.5)$ & $41(22.3)$ & \\
\hline Age, mean (SD) & $30.8(16.2)$ & $24.3(13.6)$ & $28.1(16.6)$ & $27.8(15.7)$ & 0.077 \\
\hline Grading & & & & & 0.231 \\
\hline Mild & $46(75.4)$ & - & $45(69.2)$ & $91(49.5)$ & \\
\hline Moderate & $12(19.7)$ & - & $11(16.9)$ & $23(12.5)$ & \\
\hline Severe & $3(4.9)$ & - & $9(13.9)$ & $12(6.5)$ & \\
\hline GOS & & & & & 0.380 \\
\hline Low disability & $12(19.7)$ & $18(31.1)$ & $12(18.5)$ & $42(22.8)$ & \\
\hline Moderate disability & $21(34.4)$ & $16(27.6)$ & $16(24.6)$ & $53(28.8)$ & \\
\hline Severe disability & $10(16.4)$ & $6(10.3)$ & $11(16.9)$ & $27(14.7)$ & \\
\hline Vegetative & $11(18.0)$ & $6(10.3)$ & $11(16.9)$ & $28(15.2)$ & \\
\hline Death & $7(11.5)$ & $12(20.7)$ & $15(23.1)$ & $34(18.5)$ & \\
\hline Admission GCS & & & & & 0.141 \\
\hline Moderate & $17(27.9)$ & $11(19.0)$ & $9(13.9)$ & $37(20.1)$ & \\
\hline Severe & $44(72.1)$ & $47(81.0)$ & $56(86.2)$ & 147 (79.9) & \\
\hline Discharge GCS & & & & & 0.195 \\
\hline Moderate & $28(82.3)$ & $15(71.4)$ & $20(62.5)$ & $63(72.4)$ & \\
\hline Severe & $6(17.7)$ & $6(28.6)$ & $12(37.5)$ & $24(27.6)$ & \\
\hline Onset (days), median (IQR) & $5(3-7)$ & - & $2(1-4)$ & $3(2-6)$ & 0.002 \\
\hline Peak (days), median (IQR) & $7(4-8)$ & - & $3(2-5)$ & $5(2-7)$ & 0.001 \\
\hline Duration (days), median (IQR) & $3(2-6)$ & - & $3(1-4)$ & $3(2-5)$ & 0.145 \\
\hline
\end{tabular}

Table 2. Sodium abnormality, n (\%)

\begin{tabular}{lccc}
\hline Hypernatremia & \multicolumn{3}{c}{ Hyponatremia } \\
\hline Dehydration & $52(80.0)$ & Fluid overload & $47(77.1)$ \\
DI & $7(10.8)$ & SIADH & $11(18.0)$ \\
Hyperosmolar therapy & $6(9.2)$ & CSW & $3(4.9)$
\end{tabular}

Table 3. TBI severity on admission, $n(\%)$

\begin{tabular}{lcc}
\hline Grade of head injury severity (GCS) & Good outcome (GOS 4-5) & Poor outcome (GOS 1-3) \\
\hline Moderate TBI (GCS) & $29(78.4)$ & $8(21.6)$ \\
Severe TBI (GCS) & $66(44.9)$ & $81(55.1)$
\end{tabular}

Abnormal serum sodium concentrations were present in $126(68.4 \%)$ patients of whom 61 (33.1\%) had hyponatremia and $65(35.3 \%)$ hypernatremia (Table 1$)$. In the hypernatremia group, $52(80 \%)$ had dehydration post resuscitation at 6 hours, $7(10.7 \%)$ had DI and $6(9.3 \%)$ had hyperosmolar therapy as the cause. In the hyponatremia group, 47 (77.1\%) had fluid overload, 11 (18\%) had SIADH and $3(4.9 \%)$ had CSWS (Table 2). Age was not associated with sodium abnormalities $(p=0.077)$ but those within the normal sodium concentration group had the lowest mean age. There was no significant correlation between mechanism of injury and sodium abnormality. The majority of patients with moderate TBI had a good outcome $(78.3 \%)$, while the majority of patients with severe TBI had a poor outcome (55.1\%) (Table 3).

The peak differed by sodium abnormality (Figure 1) with $50 \%$ of the hyponatremia patients peaking 4 days later than the hypernatremia patients $(\mathrm{p}=0.001)$. Hypernatremia peaked at day 3 (IQR 2-5) and hyponatremia troughed at day 7 (IQR 4-8). The analysis of the onset of sodium abnormality showed that for those patients who survived, a later onset was related to a better outcome $(p=0.024)$. There was no correlation 


\begin{tabular}{lc} 
Table 4. CT findings on admission, $\mathbf{n}(\%)$ & \\
\hline Diffuse brain injury & $73(39.7)$ \\
Cerebral contusions & $30(16.3)$ \\
Acute subdural haematoma & $28(15.2)$ \\
Traumatic subarachnoid haemorrhage & $9(4.9)$ \\
Intraventricular haemorrhage & $4(2.2)$ \\
Acute subdural haematoma with contusions & $10(5.4)$ \\
Acute extradural haematoma & $11(6)$ \\
Brainstem elongation & $1(0.5)$ \\
Tract haematoma & $3(1.6)$ \\
Intracerebral haematoma & $2(1.1)$ \\
Subacute subdural haematoma & $1(0.5)$ \\
Posterior fossa extradural haematoma & $1(0.5)$ \\
Comminuted skull fracture & $7(4)$ \\
Posterior fossa contusion & $1(0.5)$ \\
Skull base fracture & $2(1.1)$ \\
Previous infarct with no new haemorrhage & $1(0.5)$
\end{tabular}

Table 5. Management, n (\%)

\begin{tabular}{lc}
\hline ICP monitor & $67(36.4 \%)$ \\
Craniotomy and an ICP monitor & $38(20.7 \%)$ \\
Decompressive craniectomy and an ICP monitor & $27(14.7 \%)$ \\
$\begin{array}{l}\text { Neuroprotective ventilation only with no ICP } \\
\text { monitor inserted }\end{array}$ & $49(26.6 \%)$ \\
Wound debridement & $2(1.1 \%)$ \\
Neurological observation & $1(0.5 \%)$
\end{tabular}

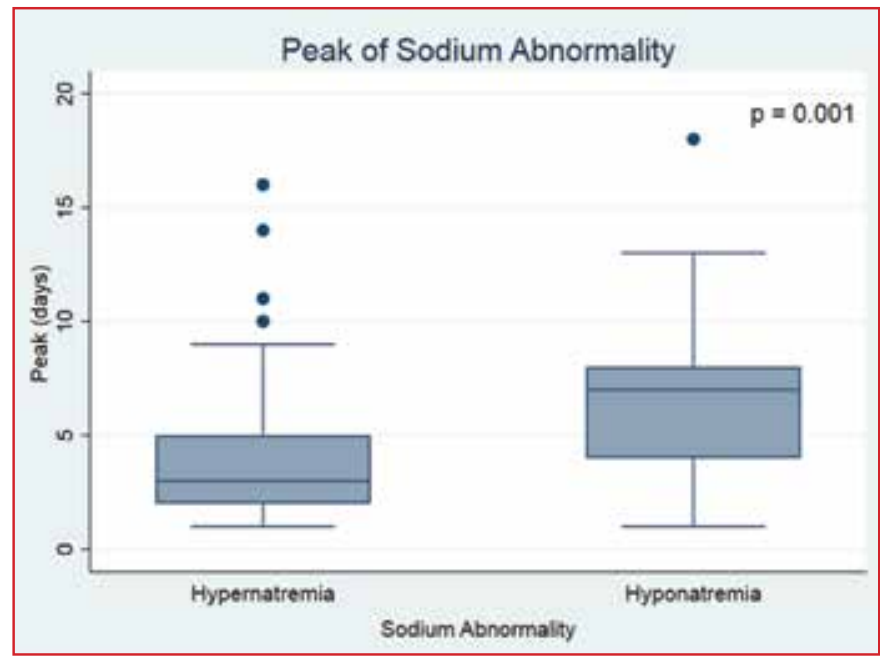

Figure 1.

between age and duration of sodium abnormality $(r=0.11$, $p=0.202)$ and duration did not differ by sodium abnormality $(\mathrm{p}=0.145)$.

Glasgow Outcome Score (GOS) results reflected that the majority of patients had either low $(22.8 \%)$ or moderate (28.8\%) disability. Although the underlying pathology of TBI was not associated with GOS $(p=0.059)$, the GOS of patients with DI tended to be poorer $(\mathrm{p}=0.031)$ ranging from severe disability (14.3\%) to death (71.4\%).
There was no association between the type of fluid used and GOS $(\mathrm{p}=0.159)$. Death occurred in $34(18.5 \%)$ patients and diagnosis was found to be significantly associated with mortality $(\mathrm{p}=0.01)$, the most common diagnoses amongst those who died being dehydration (29.4\%), fluid overload $(17.7 \%)$ and DI (14.7\%). Of those patients who survived, 133 (88.7\%) had improved GCS post intervention whilst $12(8 \%)$ experienced a decreased GCS and $5(3.3 \%)$ patients remained unchanged. GCS on discharge was significantly higher than GCS on admission within each sodium abnormality $(\mathrm{p}<0.0001)$.

The predominant fluid in adults was plasmalyte in $57.7 \%$, Ringer's lactate $22.5 \%$ and normal saline $8.2 \%$. The predominant fluid in paediatrics was paediatric maintenance solution. There was no association between fluid used and $\operatorname{GOS}(\mathrm{p}=0.159)$.

\section{Discussion}

In this study, abnormal serum sodium concentrations were present in $126(68.4 \%)$ patients with a prevalence of $61(33.1 \%)$ cases of hyponatremia and 65 (35.3\%) hypernatremia. In a retrospective cohort study, Maggiore et al. ${ }^{1}$ verified that hypernatremia was common, occurring in $51.5 \%$ of patients for $31 \%$ of the duration of the ICU stay. The prevalence of sodium abnormalities in this study was comparable to previous studies, ${ }^{1-3}$ however it is the first analysis of this nature in KwaZulu-Natal.

In this study patients with hypernatremia had a worse outcome than those with hyponatremia, with $21 \%$ of patients who died having associated DI. This will often occur as brain death occurs in severe TBI and consequently terminal DI may be more part of the occurrence of brain death in these patients. Severe hypernatremia following TBI is due to damage to the hypothalamic-pituitary axis and decreased production of antidiuretic hormone $(\mathrm{ADH})$ resulting in diabetes insipidus (DI). Although lesions may occur at any point along this axis, the most common aetiology is pituitary gland dysfunction as a result of swelling, ischaemia, or direct injury in those with basal skull fractures. This pathology causes rapid fluid depletion and escalation of the serum sodium levels to moderate and severe derangements. It has been recognised that this causes secondary cerebral oedema during rapid fluid replacement, resulting in deterioration of patients and was possibly the reason for its correlation with outcome. ${ }^{11,12,14}$ Neurological dysfunction is the principal manifestation of hypernatremia especially in patients with intracranial pathology. ${ }^{5,6}$ Hypernatremia was associated with a threefold increase in ICU mortality in one study. ${ }^{3}$ This study confirms a poor outcome as defined by the GOS in patients with hypernatremia as the GOS of patients with DI tended to be poorer ranging from severe disability to death.

The current study found that the maximum abnormality in serum sodium differed by sodium abnormality with $50 \%$ of the hyponatremia patients peaking 4 days later than the hypernatremia patients. The analysis of the onset of sodium abnormality showed that for those patients who survived, 
a later onset was related to a better outcome. Meticulous attention should be paid to repetitive and accurate checking of serum sodium concentration especially within the first ten days of TBI. ${ }^{2}$ The mean time after which the disturbance occurred was 23 days after head trauma in one study. ${ }^{2}$ Hyponatremia is a common neuro-medical problem seen in survivors of central nervous system injury. ${ }^{7-10}$ It is associated with seizures, coma, persistent vegetative state and brainstem compression and death as complications. ${ }^{3,13}$ It was found, in a previous study, that $20 \%$ of patients suffered an acute onset of severe hyponatremia following TBI and this was associated with a poor neurological outcome or death after sudden onset of seizures, followed by coma, apnoea and brainstem compression. ${ }^{3}$ This highlights the importance of detecting and correcting hyponatremia as soon as possible.

There was no significant correlation between mechanism of injury and sodium abnormality. The majority of patients with moderate TBI had a good outcome $(78.3 \%)$, while majority of patients with severe TBI had a poor outcome $(55.1 \%)$. Paiva et al. ${ }^{3}$ could find no association among various types of TBI and the proportion of sodium disorders, although the incidence of sodium disorders was greater in those suffering diffuse injury compared to intracerebral haematomas.

The current study found a high prevalence of dehydration and fluid overload in our patient cohort. It is possible that the high incidence of dehydration is secondary to patients being poorly resuscitated at referral centres, while the high incidence of fluid overload is possibly due to the overzealous resuscitation of some patients with additional aggressive resuscitation at referral centres. There are currently no statistical data to support this, however and a future study will be helpful to determine what percentage of patients are under-resuscitated or over-resuscitated from referral centres. There exists currently no ideal device to adequately assess a ventilated trauma patient's fluid volume to guide resuscitation and current diagnostic techniques such as IVC compressibility, pulmonary wedge catheter and stroke volume variation are not accurate enough to be more than a guide to resuscitation in any centre. It is vital that fluid management of these patients with moderate to severe TBI be optimised to prevent sodium flux, either upward or downward. To further decrease the risk of sodium abnormalities a suitable fluid management protocol must be developed for implementation both at the initial resuscitation hospital and in the ongoing management of these patients.

Age was not associated with sodium abnormalities but those within the normal sodium concentration group had the lowest mean age. A previous study suggested that most of the patients with sodium abnormalities were 21-50-year-old males who had undergone craniotomy. ${ }^{2}$

GOS at discharge reflected that the majority of patients had either low $(22.8 \%)$ or moderate $(28.8 \%)$ disability. There was no association between the type of fluid used and GOS. Death occurred in $34(18.5 \%)$ patients and diagnosis was found to be significantly associated with mortality, the most common diagnoses amongst those who died being dehydration $(29.4 \%)$, fluid overload (17.7\%) and DI (14.7\%). The GCS has been reported to correlate with sodium levels where a lower GCS was associated with a lower sodium level. ${ }^{4}$ The GOS however was not discussed in that study.

The predominant intravenous fluid in adults was plasmalyte in $57.7 \%$, Ringer's lactate $22.5 \%$ and normal saline $8.2 \%$. The predominant fluid in paediatrics was paediatric maintenance solution. There was no association between fluid used and GOS.

\section{Limitations}

The majority of our neurosurgical trauma patients are not followed up and the long-term outcome could not be assessed. The fluids given at referral centres cannot be controlled and may not have been clearly documented on referral letters to the unit on admission. Patients are not routinely taken directly to the trauma unit and this allows influence from referral centres on the fluid given to patients.

\section{Conclusion}

The prevalence of abnormal serum sodium concentrations was almost $69 \%$ of whom almost half had hyponatremia and the rest hypernatremia. Death occurred in 34 (18.5\%) patients and among those who died dehydration (29.4\%), fluid overload (17.7\%) and DI (14.7\%) were significantly associated with mortality. The analysis of the onset of sodium abnormality showed that for those patients who survived, a later onset was related to a better outcome. This makes early diagnosis of abnormal serum sodium important to identify and treat the underlying cause. An early screening tool should be in place in all critical care units where moderate to severe TBI is managed to establish if serum sodium is abnormal, for example, regular blood gas measurements with electrolytes. A controlled fluid management protocol is required at both referral hospitals and in the ongoing management of these patients with moderate to severe TBI to reduce the prevalence of sodium abnormalities.

\section{Disclosure}

The authors report no conflict of interest in this study.

\section{REFERENCES}

1. Maggiore U, Picetti E, Antonucci E, et al. The relation between the incidence of hypernatremia and mortality in patients with severe traumatic brain injury. Crit Care. 2009;13(4):R110. doi:10.1186/cc7953 [PMID:19583864]

2. Safavi, M, Abrishamkar S, Tvakoli P, Moradi D, Honarmand A. Predisposing factors for serum sodium disturbance in patients with severe traumatic brain injury (SBI). Turk J Med Sci, 2010;40(6), 851-5. doi: http://dx.doi.org/10.3906/sag-0908-202

3. Paiva WS, Bezerra DAF, Amorim RLO, et al. Serum sodium disorders in patients with traumatic brain injury. Ther Clin Risk Manag. 2011;7:345. doi:10.2147/TCRM.S17692 [PMID:21941440]

4. Adiga US, Vickneshwaran V, Sen SK. Electrolyte derangements in traumatic brain injury. Basic Research Journal of Medicine 
and Clinical Sciences. 2012;1(2):15-8. [ISSN, 2315-6864]

5. John CCA, Day MW. Central neurogenic diabetes insipidus, syndrome of inappropriate secretion of antidiuretic hormone, and cerebral salt-wasting syndrome in traumatic brain injury. Crit Care Nurse. 2012;32(2):e1-e7. doi:10.4037/ccn2012904 [PMID:22467619]

6. Wilson JR, Green A. Acute traumatic brain injury: a review of recent advances in imaging and management. Eur J Trauma Emerg Surg. 1 Apr 2009;35(2):176. doi: https://doi.org/10.1007/ s00068-008 -8095-8 [ ISSN 1863-9941]

7. Zafonte RD, Mann NR. Cerebral salt wasting syndrome in brain injury patients: a potential cause of hyponatremia. Arch Phys Med Rehabil. 1997;78(5):540-2. [PMID:9161378]

8. Moro N, Katayama Y, Igarashi T, Mori T, Kawamata T, Kojima J. Hyponatremia in patients with traumatic brain injury: incidence, mechanism, and response to sodium supplementation or retention therapy with hydrocortisone. Surg Neurol. 2007;68(4):387-93. doi:10.1016/j.surneu.2006.11.052 [ PMID:17905062]

9. Costa KN, Nakamura HM, Cruz LRD, et al. Hyponatremia and brain injury: absence of alterations of serum brain natriuretic peptide and vasopressin. Arq Neuropsiquiatr. 2009;67(4):103744. doi: http://dx.doi.org/10.1590/S0004-282X2009000600014
[ISSN 0004-282X]

10. Kirkman MA, Albert AF, Ibrahim A, Doberenz D. Hyponatremia and brain injury: historical and contemporary perspectives. Neurocrit Care. 2013;18(3):406-16. doi:10.1007/s12028-0129805-y [PMID:23212244]

11. Tommasino C. Fluids and the neurosurgical patient. Anesthesiol Clin North America. 1 Jun 2002;20(2):329-46. doi: 10.1016/ S0889-8537(01)00013-X [PMID:12165997]

12. Wright WL. Sodium and fluid management in acute brain injury. Curr Neurol Neurosci Rep. Aug 2012;12(4):466-73. doi: 10.1007/s11910-012-0284-5 [PMID 22622407]

13. Zygun DA. Sodium and brain injury: do we know what we are doing? Crit Care. Oct 2009;13(5):184. doi: 10.1186/cc8014 [PMID 19804616]

14. Bradshaw K, Smith M, Disorders of sodium balance after brain injury. BJA Educ Continuing Education in Anaesthesia Critical Care \& Pain. 31 Aug 2008;8(4):129-33. doi: 10.1093/bjaceaccp/ mkn019

15. O'Donoghue SD, Dulhunty JM, Bandeshe HK. Acquired hypernatraemia is an independent predictor of mortality in critically ill patients. Anaesthesia. May 2009;64(5):514-20. doi: 10.1111/j.1365-2044.2008. 05857.x [PMID 19413821] 